bedarf gegeben sowie einen beachtlichen Frauengesundheitsbericht, der unter der Federführung des BMFSFJ erstellt wurde. Daneben und danach gab es eine Vielzahl weiterer Untersuchungen und Analysen, deren Ergebnisse stets dasselbe sagen: zielführende medizinische Versorgung setzt einen konsequenten geschlechtsspezifischen Ansatz voraus. Umso unverständlicher, dass dieser bis heute keinen Eingang in die gesundheitspolitische Programmatik gefunden hat.

Auch haben sämtliche Gesundheitsreformen keinerlei Ansätze für eine Institutionalisierung der Geschlechterparität in den Gremien gezeigt. Besonders unverständlich ist der fehlende Gender-Ansatz bei der angestrebten Straffung und transparenteren Ausgestaltung der Gremienarbeit: So sinnvoll es ist, die Entscheidungen über neue Untersuchungs- und Behandlungsmethoden in angemessener Frist zu treffen, so wichtig ist es festzuschreiben, dass wesentliche Verfahrensvoraussetzung eine klare geschlechtersensible Darstellung des Verfahrens ist.

Seit 2003 rechnen die Krankenhäuser mit den Krankenkassen nach Fallpauschalen, den sogenannten Diagnosis Related Groups - DRG - ab. Diese richten sich nicht nach der Länge des Krankenhausaufenthaltes, sondern nach Diagnosen und den durchgeführten Maßnahmen. Insgesamt führten sie zu einer Reduzierung der Liegezeiten. Dieses führt dazu, dass die ambulante Nachsorge durch niedergelassene Fachärzte und Sozialstationen erfolgen soll. De facto sind jedoch die Budgets der niedergelassenen Vertragsärzte so klein, dass selbst bei bestem Wissen und Wollen eine aufwendige postoperative häusliche Betreuung unmöglich ist. Gleiches gilt für die pflegerische Versorgung, ganz zu schweigen von so praktischen Problemen, wie denn Hilfskräfte alarmiert werden können, und wie sie dann z. B. in die Wohnung kommen, wenn Patienten nicht aufstehen können. Wer hilft beim Toilettengang? Wer kauft ein, wer bringt einem gehbehinderten Patienten Getränke und Essen? Wer wäscht? Wer versorgt Kinder, Haustiere etc.? Für junge und mittelalte Patienten ist all dies bereits heute zum Teil unlösbar, besonders wenn es sich um Singles handelt, die im 5. Stock ohne Fahrstuhl oder in einer mehrstöckigen Maisonettewohnung wohnen. In diesen Fällen wird die fehlende häuslichen Versorgung bereits heute deutlich. Bei früherer Entlassung nach Operationen an den Beinen oder gar ambulanten Operationen dürfte hier mit einem deutlichen Anstieg von Komplikationen gerechnet werden.

Die Folge ist: Wenn Liegezeiten im Krankenhaus verkürzt werden, müssen „krankheitsgerechte“ Wohnungen mit familienähnlichen Versorgungsstrukturen - ergänzt um Ärztinnen und Ärzte, Pflegekräfte und Physiotherapeutinnen und Physiotherapeuten - also eigentlich Krankenhäuser im ursprünglichen Sinn angeboten werden. Kinderversorgung in der Wohnung wurde ebenso wie Pflege von Angehörigen früher von Haushalts- und Hofhilfen oder Gemeindeschwestern übernommen, die auf Zeit in die Wohnung mit einzogen oder tagsüber dort blieben, bis Angehörige von der Arbeit kamen und den Haushalt wieder übernahmen. Diese Strukturen sind außer in wenigen ländlichen Regionen - weggebrochen und es gibt hier weder Personal noch Geld, um sie wieder aufzubauen.

Zur Gesundheit gehören neben der Eigenverantwortung und der solidarischen Krankenversorgung auch Rahmenbedingungen für eine gesunde Lebensführung. Diese werden von allen Politikbereichen gestaltet. Investitionen in gute Rahmenbedingungen tragen wesentlich zur Kostensenkung im Gesundheitswesen bei. Fehlende und schlecht bezahlte Arbeit, zerstörte Umwelt, industriell und mit fragwürdigen Ingredienzen produzierte Nahrung und nicht zuletzt fehlende passende Kinderbetreuung können krank machen. Wie soll sich eine Versicherte oder ein Versicherter sportlich ertüchtigen, wenn die kommunalen Bäder reihenweise aus Kostengründen geschlossen werden? Wie sollen Kinder an eine gesunde Lebensweise herangeführt werden, wenn Schulen das Thema nicht aufgreifen?

Eine Gesundheitsreform, die diesen Namen verdient, muss die unbestreitbar positiven Seiten der GKV stärken, eine breitere Einnahmenseite schaffen und für alle - auch für Frauen und Familien - eine bezahlbare Sicherung ihrer Gesundheit bieten.

\title{
Pflege als staatliche Aufgabe
}

Der demografische Wandel erfordert zunehmende Anstrengungen bei der Pflege und ihrer sozialen Absicherung. Die steigende Zahl hochbetagter Menschen ist ein erfreulicher Effekt moderner Medizin. Doch wird dadurch zugleich die Gewährleistung und Finanzierung von Pflege zu einer schwierigen politischen Aufgabe. Der djb übernimmt eine besondere Verantwortung für die rechtlichen Grundlagen der Pflege. Sein Satzungszweck gebietet ihm die Sorge nicht nur für die Angelegenheiten der Frauen, sondern auch für die der älteren Men- schen. Auf dem Gebiet der Pflege bilden diese Gruppen eine große Schnittmenge.

Der djb bemüht sich seit Einführung der Pflegeversicherung um eine geschlechter- und familiengerechte Ausgestaltung des Beitragsrechts. Hier besteht auch gegenwärtig gesetzlicher Nachbesserungsbedarf. Das Bundesverfassungsgericht forderte 2001 eine familiengerechte Beitragsgestaltung, die Familien nicht erst in Zukunft, sondern bereits zum Zeitpunkt der Beitragszahlung entlasten sollte. ${ }^{1}$ Der Gesetzgeber ent- 
schied sich 2004 für eine Erhöhung des Beitragssatzes für „Kinderlose“, 2 die keineswegs gerecht ausgestaltet wurde. ${ }^{3}$ Von der gegenwärtigen Beitragsgestaltung profitieren „Eltern“ nämlich unabhängig von der Zahl der Kinder und ohne Rücksicht darauf, ob sie überhaupt noch Kinder im erziehungsund betreuungsbedürftigen Alter haben. Außerdem ist nicht gewährleistet, dass die freiwerdenden Mittel für die Altersvorsorge gerade des erziehenden und betreuenden Elternteils - in der Regel der Mutter - aufgewandt werden. Dem Anliegen des Bundesverfassungsgerichts, Mittel für erziehende und betreuende Eltern für die weitere Altersvorsorge freizustellen, entspricht eine solche Ausgestaltung nicht.

Das Augenmerk ist aber auch auf die Organisation der Pflege als persönliche (Dienst-)Leistung gerichtet. Mit der jüngsten Pflegeversicherungsreform ${ }^{4}$ betonte und forderte die Politik erneut die Verantwortung der Familien für die Pflege ihrer nahen Angehörigen. Da immer noch vornehmlich Töchter und Schwiegertöchter die unentgeltliche Pflege der Eltern übernehmen, nimmt die Politik ohne dies auszusprechen - besonders die Frauen in die Pflicht. Pflegeversicherungsrechtliche Komponenten, die der Entlastung und sozialen Absicherung von nicht erwerbsmäßigen Pflegepersonen gelten, sind deshalb auch gleichstellungsrechtlich relevant. In der jüngsten Pflegeversicherungsreform ist mit der unentgeltlichen Pflegezeit eine Möglichkeit geschaffen worden, Pflegearbeit und Berufstätigkeit sinnvoll miteinander zu verbinden. $\mathrm{Ob}$ die Pflegezeit in ihrer gegenwärtigen Ausgestaltung diese Vereinbarkeit bereits in ausreichendem Maße gewährleistet, muss beobachtet werden. Ganz allgemein ist Sorge dafür zu tragen, dass nicht mit zunehmender Verschiebung der Altersstrukturen und zunehmender Berufstätigkeit von Frauen die private Pflege von Angehörigen in der Familie die Rollenteilung zwischen Frauen und Männern befestigt und zu einer überproportionalen Belastung von Frauen führt.

Verstärkte politische Bedeutung kommt auch dem Sektor der Pflege- und haushaltsnahen Dienstleistungsberufe zu. In diesem Sektor sind ganz überwiegend Frauen beschäftigt. Die Politik muss hier für eine geschlechtergerechte Entlohnung Sorge tragen und „prekäre“ Arbeitsbedingungen vermeiden helfen. Da gerade im hauswirtschaftlichen Sektor häufig Personen anderer Staatsangehörigkeit beschäftigt werden, sind die erforderlichen Anstrengungen im Arbeits- und Sozialrecht um vernünftige Zuwanderungsregeln zu ergänzen.

Pflegeleistungen sollen allen Pflegebedürftigen in ausreichendem, ein menschenwürdiges Dasein si- cherndem Maß zustehen. Damit dies geleistet und finanziert werden kann, müssen Pflegeleistungen effizient organisiert und muss das benötigte Maß an Pflegeleistungen definiert werden. Diese Festlegungen erfordern Wertungen und allokative Entscheidungen der Politik, die angesichts knapper öffentlicher Kassen verstärkt zu beobachten und $\mathrm{zu}$ überprüfen sind. Auch hier ergibt sich ein gleichstellungspolitischer Akzent, weil wegen des höheren durchschnittlichen Lebensalters mehr Frauen als Männer nicht von ihren Ehe- und Lebenspartnern gepflegt werden können und auf andere Pflegeleistungen angewiesen sind. Darüber hinaus bedarf auch die freie Entscheidung gegen ein menschenunwürdiges Weiterleben der rechtlichen Absicherung. Die Regelung der Patientenverfügung ist in dieser Legislaturperiode gerade noch gelungen. ${ }^{5}$ Auch der djb hatte sich diesem Thema fortlaufend und intensiv gewidmet. ${ }^{6}$ Er wird die politischen Bemühungen zur Gestaltung der Pflege am Lebensende auch weiterhin konstruktiv und kritisch begleiten.

1 BVerfGE 103, 242 (264 ff.).

2 Gesetz zur Berücksichtigung der Kindererziehung im Beitragsrecht der sozialen Pflegeversicherung (KinderBerücksichtigungsgesetz - KiBG) vom 15.12.2004, BGBI. I, 3488.

3 Vgl. demgegenüber die Stellungnahme des djb „Berücksichtigung des Aufziehens von Kindern in der Pflegeversicherung: djb-Vorschlag zur Umsetzung der Pflegeversicherungs-Entscheidungen des Bundesverfassungsgerichts (BVerfG) vom 3. April 2001" vom 10.6.2004, abrufbar unter www.djb.de/ stellungnahmen-und-pressemitteilungen/ stellungnahmen/2004.html.

4 Gesetz zur strukturellen Weiterentwicklung der Pflegeversicherung (Pflege-Weiterentwicklungsgesetz) vom 28.5.2008, BGBI. I, 874 .

5 Drittes Gesetz zur Änderung des Betreuungsrechts vom 29.7.2009, BGBI. 2009 I, 2286.

6 Vgl. zuletzt Stellungnahme des djb zu verschiedenen Entwürfen zu einer gesetzlichen Regelung der Patientenverfügung anlässlich der öffentlichen Anhörung vor dem Rechtsausschuss des Bundestages am 4.3.2009, abrufbar unter www.djb.de/stellungnahmen-und-pressemitteilungen/ stellungnahmen/2009.html.
Prof. Dr. Margarete Schuler-Harms

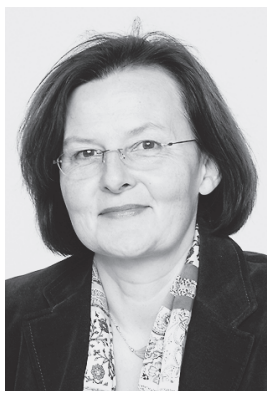

Mitglied der Kommission Recht der sozialen Sicherung, Familienlastenausgleich des djb; Universität der Bundeswehr, Hamburg 